\title{
Does a BIS-Guided Maintenance of Anesthetic Depth Prevent Implicit Memory?
}

\author{
Mehmet Sertac Ozcan ${ }^{1}$, Scott Douglas Gronlund ${ }^{2}$, Ryan Trojan ${ }^{3}$, Qaiser Khan ${ }^{3}$, \\ Jorge Cure ${ }^{3}$, Carson Wong ${ }^{4}$ \\ ${ }^{1}$ Department of Anesthesiology, College of Medicine, University of Illinois at Chicago, Chicago, USA; \\ ${ }^{2}$ Department of Psychology, College of Arts and Sciences, the University of Oklahoma, Norman, USA; \\ ${ }^{3}$ Department of Anesthesiology, College of Medicine, the University of Oklahoma Health Sciences Cen- \\ ter, Oklahoma City, USA; \\ ${ }^{4}$ Department of Urology, College of Medicine, the University of Oklahoma Health Sciences Center, \\ Oklahoma City, USA. \\ Email: msozcan@gmail.com \\ Received January $6^{\text {th }}, 2011$; revised February $28^{\text {th }}$, 2011; accepted April $1^{\text {st }}, 2011$.
}

\begin{abstract}
Recent studies investigating the relationship between depth of anesthesia and implicit memory have conflicting results. Limitations of these studies include lack of standardization in surgical procedures and failure to control depth of anesthesia prospectively. We assessed implicit memory function at two different (BIS-guided and prospectively controlled) anesthetic depths during surgical stimulus. A list of words was played via headphones to 37 patients during orthopedic surgery under general anesthesia. The Bispectral (BIS) Index was monitored and patients were randomized to remain in a deeper (BIS 40 - 45) or lighter (BIS 55 - 60) plane of surgical anesthesia during word presentation. Postoperatively, implicit memory performance was tested using a simple auditory word-stem completion test for presented as well as non-presented words. Absence of explicit memory was evaluated by asking four standard questions regarding intraoperative awareness. All patients received sevoflurane and fentanyl for general anesthesia. There was no evidence of implicit memory in either study group. Hit rates for presented and non-presented words were $0.23 \pm 0.14$ and $0.25 \pm 0.09$, respectively. No explicit memory was reported by any patient. Depth of anesthesia did not affect implicit memory formation in anesthetized patients undergoing surgery. General anesthesia, even at a higher BIS range, appears to abolish implicit memory as assessed by a simple word-stem completion test.
\end{abstract}

Keywords: Implicit Memory, General Anesthesia, Depth of Anesthesia

\section{Introduction}

Memory during anesthesia continues to be an interesting subject for anesthesiologists and psychologists alike. A review of the recent literature suggests a continuing interest in the subject in the last decade (e.g., Andrade \& Deeprose, 2007). This coincides with recent advances in both anesthesiology and cognitive psychology. Depth of anesthesia monitors such as the Bispectral Index (BIS) are being increasingly used in clinical practice and as well as in memory studies (Bowdle, 2006). On the psychology front, Schacter (1987) has classified episodic memory into explicit (or conscious) and implicit (or unconscious) types. These advances inspired investigators to ask whether explicit and implicit memory might be preserved under general anesthesia.

BIS is a patient monitor that quantifies the depth of the hypnotic component of general anesthesia (Bowdle, 2006). It uses electroencephalogram recorded over the forehead and computes a number (ranging from 0 to 99) using a proprietary algorithm. This number is displayed on the monitor during an anesthetic and it can help the anesthesiologist to titrate the anesthetic agents. A BIS range of 40 to 60 has generally been accepted to represent an ideal anesthetic depth of where explicit recall is extremely unlikely (Bowdle, 2006).

Formation of explicit memories during general anesthesia is associated with intraoperative awareness. This has been recog- nized since the early days of clinical anesthesia practice (Wright \& Aldrete, 1987). In one survey (Stoelting, 1999), more than one third of anesthesiologists ranked intraoperative awareness as a major patient-safety concern. It has been associated with morbidity such as post-traumatic stress disorder (Sandin, 2006). Implicit memory during anesthesia has been recognized more recently. Because there is no conscious recollection of implicit memories, they can only be demonstrated by special tests. Presenting a list of spoken words (priming) under anesthesia and subsequent administration of a word-stem completion (WSC) test after emergence from anesthesia is a common method used for this purpose. In WSC, subjects are asked to complete the word stem with the first word that comes to mind. The WSC test has been demonstrated as a sensitive method of identifying implicit memory under anesthesia (Andrade \& Deeprose, 2007; Deeprose, Andrade, Varma, \& Edwards, 2004).

Alternatively, a process dissociation procedure (PDP) can be used to detect and eliminate the contribution of explicit memory from true implicit memory effects (e.g., Jacoby, 1991). PDP has inclusion and exclusion conditions. The inclusion condition requires the subject to complete the word-stem with either a word that was recalled from the intraoperative period, or with the first word that comes to mind if there is no recall of a word heard intraoperatively. In contrast, the exclusion condition requires the subject to not complete the word-stem with a word that can be recalled from the intraoperative period and instead 
come up with another word that completes the stem. That is, the subject excludes consciously recalled words. Simple algebraic manipulations and subtractions result in estimates for the explicit and implicit memory contributions (given the contentious assumption of independence between these contributions). Although using PDP purportedly increases the specificity of detecting implicit memory effects, it is more complex, makes controversial assumptions, and is awkward for the patient during the perioperative period because the patient is asked to try to recall a word from a period when they report not recalling anything. Besides these concerns, PDP as a method for detecting intraoperative implicit memory has been questioned for potentially generating false positive results (Hadzidiakos et al., 2009).

Implicit memory formation without explicit recall during general anesthesia has been demonstrated in a number of studies (e.g., Deeprose et al., 2009; Dobrunz, Jaeger, \& Vetter, 2007; Iselin-Chaves et al, 2005; Lubke, Kerssens, Phaf, \& Sebel, 1999; Stonell, Leslie, He, \& Lee, 2006). However, there are other studies in which these results were not replicated (e.g., Kerssens, Ouchi, \& Sebel, 2005; Lequeux et al., 2005; Lequeux, Cantraine, Levarlet, \& Barvais, 2003; Munte et al. 2001; Russell \& Wang, 2001). A review of these studies suggests that depth of anesthesia and the presence of surgical stimulus as factors that might modulate implicit memory formation. However, there are conflicting findings regarding both of these potential factors. For instance, Stonell et al. reported that implicit memory was present only when the BIS was greater than 50 . Iselin-Chaves et al. demonstrated that the incidence of implicit memory was similar at BIS ranges of 41 - 60 and 61 - 80. On the other hand, Kerssens et al. found no evidence of implicit memory when BIS was between 40 and 60 during word priming. Unfortunately, only some of those studies conducted the word priming procedure during surgical stimulus. Moreover, the type of surgery varied (Iselin-Chaves et al., 2005; Kerssens et al., 2005) or was not reported (Stonell et al., 2006). In addition, most of these articles did not specify whether the word priming coincided with a consistently painful period during a surgical procedure (e.g., immediately after incision) in all patients. In short, factors facilitating or preventing the formation of implicit memory during general anesthesia are not clear from the available data, because few studies effectively and prospectively controlled these potential factors.

The aim of the present study was to investigate the effect of depth of anesthesia on formation of implicit memory during orthopedic procedures. We hypothesized that implicit memory might be better preserved at a lighter plane of anesthesia compared to a deeper anesthetic plane. To test our hypothesis, we designed a study in which depth of anesthesia was prospectively controlled and maintained in patients randomized into two groups (BIS ranges of 55 to 60 and 40 to 45). A list of auditory words was presented at the same phase of the procedure (i.e., immediately starting with surgical instrumentation of the bone) in all subjects. After emergence from anesthesia, a word-stem completion (WSC) test was conducted to detect implicit memory.

\section{Method}

\section{Participants}

An Institutional Review Board at the University of Okla- homa Health Sciences Center approved the study before any consent was obtained. All patients signed an informed consent before participating. Inclusion criteria included being a literate, native English speaker and having been scheduled to have an orthopedic procedure. Patients with hearing impairment, history of cognitive dysfunction, or alcohol or other recreational drug abuse within the previous six months were excluded.

Sixty-four subjects were enrolled upon meeting the inclusion criteria but 9 of those were dropped from the study before any study intervention was made (surgery was cancelled in three cases, two patients decided not to proceed with the study, study equipment was unavailable in two cases, and exclusion criteria were discovered on the day of surgery in two other cases). Exposure phase was completed in 53 subjects. Subsequently, seven subjects were excluded from the BIS 55 - 60 group due to inability to maintain BIS within the target range (average BIS during word priming were $<50$ in these seven subjects). Four subjects were excluded from BIS 40 - 45 group, one of whom remained intubated for three days postoperatively, another was too sedated to complete the test phase, and the remaining two had unacceptably poor audio quality of the recorded WSC test. Data from the remaining 37 subjects were included in the final analysis, 20 of which were in the BIS 40 - 45 group and 17 in the BIS 55 - 60 group.

Table 1 shows patient demographics, distribution of surgical procedures, and intraoperative variables. Demographic variables were analyzed using independent sample $t$-tests. There were no significant differences in demographic variables between the two groups.

Patients admitted to the hospital postoperatively were tested in the morning of the first post-operative day. Others that had a same-day procedure were tested upon recovery from anesthesia, immediately before their discharge from the hospital. Table 2 details the timing of the WSC test in relation to priming, emergence from anesthesia, and the day of surgery. The ratio of patients who had the WSC test on the day of surgery was 35\% in both groups.

\section{Anesthetic Technique}

All patients were premedicated with $2 \mathrm{mg}$ IV midazolam immediately before being transferred to the operating room. Standard anesthesia monitors (including EKG, non-invasive blood pressure, pulse oximetry, temperature, and end-tidal gas monitoring) were applied in the operating room. Bispectral index (BIS) (S/5 BIS module, DSC XP, Datex-Ohmeda, Helsinki, Finland) was monitored in all patients starting from before the induction until the end of the intraoperative study procedure.

A bolus of fentanyl (2 g. $\left.\mathrm{kg}^{-1}\right)$ was given intravenously immediately before preoxygenation. After preoxygenation for two minutes, anesthesia was induced with propofol (1.5 to $2 \mathrm{mg}$. $\mathrm{kg}^{-1}$ ) in a dose sufficient to abolish the eyelash reflex. Suxamethonium (1 mg. $\left.\mathrm{kg}^{-1}\right)$ or cis-atracurium $\left(0.15 \mathrm{mg}^{\mathrm{kg}} \mathrm{kg}^{-1}\right)$ was used to facilitate tracheal intubation. Anesthesia was maintained with sevoflurane and air 50\% in oxygen. Ventilation was controlled artificially to attain an end-tidal partial pressure of carbon dioxide of 33 to $38 \mathrm{mmHg}$.

Immediately following tracheal intubation, patients were randomly assigned into one of two groups: maintenance of anesthesia in a lighter (BIS range of 55 to 60) or deeper (BIS 
Table 1.

Demographics and Clinical Variables.

\begin{tabular}{|c|c|c|c|}
\hline & $\begin{array}{c}\text { BIS } 40-45 \\
\quad(n=20)\end{array}$ & $\begin{array}{c}\text { BIS } 55-60 \\
\quad(n=17)\end{array}$ & $p$ \\
\hline Age (years) & $43 \pm 14$ & $43 \pm 14$ & 1.0 \\
\hline Gender (Male/Female) & $14 / 6$ & $10 / 7$ & \\
\hline Duration of Anesthesia (min) & $303 \pm 134$ & $297 \pm 123$ & 0.89 \\
\hline Duration of Surgery (min) & $231 \pm 120$ & $222 \pm 102$ & 0.81 \\
\hline BIS during word presentation & $39 \pm 5$ & $55 \pm 7$ & $<0.001$ \\
\hline etSev during word presentation (\%) & $1.5 \pm 0.4$ & $1.3 \pm 0.4$ & 0.14 \\
\hline \multicolumn{4}{|l|}{ Fentanyl dose } \\
\hline Until word presentation completed & $75 \pm 83$ & $198 \pm 111$ & $<0.001$ \\
\hline Total & $225 \pm 155$ & $308 \pm 114$ & 0.08 \\
\hline Mean BP during word presentation (mmHg) & $85 \pm 16$ & $90 \pm 15$ & 0.34 \\
\hline
\end{tabular}

etSev: end-tidal Sevoflurane; BP: blood pressure. Data are presented as mean \pm standard deviation and compared using a $t$-test. $p<0.05$ was considered statistically significant.

Table 2.

Timing of the WSC Test in Relation to Priming, Emergence from Anesthesia, and Day of Surgery.

\begin{tabular}{|c|c|c|c|}
\hline & $\begin{array}{c}\text { BIS } 40-45 \\
\quad(n=20)\end{array}$ & $\begin{array}{c}\text { BIS } 55-60 \\
\quad(n=17)\end{array}$ & $p$ \\
\hline Word Presentation to WSC test (hours) & $22.2(2.6-27.1)$ & $19.9(2-28)$ & 0.60 \\
\hline Emergence to WSC test (hours) & $20.5(1.4-26.4)$ & $17(1-26.5)$ & 0.27 \\
\hline \multicolumn{4}{|l|}{ Timing of WSC testing } \\
\hline Same Day of Surgery & $35 \%$ & $35 \%$ & \\
\hline Next day of Surgery & $65 \%$ & $65 \%$ & \\
\hline
\end{tabular}

WSC: Word stem completion. Data are presented as medians (range) and compared using a Mann-Whitney Rank Sum test.

range of 40 to 45 ) plane. BIS values were closely monitored and sevoflurane vaporizer output was continually adjusted to maintain the target BIS range. Additional fentanyl was given during surgery to provide analgesia at the discretion of the attending anesthesiologist. Additional cis-atracurium was used to prevent movement as indicated.

Amount of opioids administered until the completion of word presentation were significantly greater in the BIS 55 - 60 group (198 \pm 111 vs $75 \pm 83, p<0.001)$. End-tidal concentrations of sevoflurane were not significantly different between the groups $(1.5 \pm 0.4$ vs. $1.3 \pm 0.4, p=0.14)$.

\section{Materials}

A pool of 80 two-syllable words was selected. All the words had limited co-articulation between syllables such that the stems, when pronounced in isolation, maintained the same pronunciation as they did within a word (e.g., MUS in MUSTARD, rather than a different pronunciation, e.g., MUS in MUSHROOM). The 80 words consisted of 40 words classified as negative and 40 words classified as neutral on the trait of emotionality (John, 1988). This was done because IselinChaves et al. (2005) suggested a potential role for the amygdala.
Deeprose et al. (2004) found implicit memory during, but not before surgical stimulation, and suggested that this might be due to the body's stress reaction and the concomitant contribution of the amygdala. We hypothesized that negative words might be more likely to be implicitly preserved than neutral words, due to the amygdala's role in emotion and memory (Phelps, 2004). The scale for emotionality was from -6 (very negative feeling) to +6 (very positive feeling), with 0 being a neutral feeling. The negative group's mean was $-2.89(\mathrm{SD}=$ $0.98)$ and the neutral group's mean was $+0.96(\mathrm{SD}=0.88)$. For each word, a number of possible two-syllable completions existed for the first syllable stem. For example, the word stem "win" has ten possible two-syllable completions (e.g., window, windy, winner, winter, etc.).

Randomization of words to conditions was accomplished in the following manner: First, 20 negative and 20 neutral words were randomly selected from the initial pool of 80 words. One patient would be presented these words while under anesthesia while a patient paired to this patient would have the other 40 words presented while under anesthesia. This ensured that each word occurred equally often in the role of a presented and an unpresented stimulus. If both of these patients were assigned to the BIS level of $40-45$, a third and fourth patient would be 
presented with the same two sets of words at the BIS level of 55 - 60. The 40 selected words were randomly ordered for each patient. However, due to a programming error, only 10 of the negative words and 10 of the neutral words actually were presented during surgery. Consequently, the remaining 10 negative and 10 neutral words were treated like not presented words in the analyses that follow.

\section{Procedure}

The experiment comprised an exposure phase (word priming) and a test phase (WSC test). The exposure phase consisted of the auditory presentation of a list of words via closed headphones. The auditory stimuli were digitized and presented under the control of the E-Prime software version 1.0 (Psychology Software Tools, Inc., Pittsburgh, PA) running on a notebook computer. Auditory presentation began after the target BIS level was achieved, and within 5 minutes of the beginning of the surgical instrumentation of bone. Each auditory file (i.e. spoken word) was 2 seconds in length and was repeated 20 times consecutively. Thus, the duration of the exposure phase (word priming) was 13 minutes and 20 seconds.

To begin the test phase, patients were asked four free-response questions to elicit evidence of explicit recollection of intraoperative events ('What is the last thing you remember before falling asleep?', 'What is the first thing you remember about waking up?', 'Did you dream while you were asleep?', and 'Did you hear any words while you were asleep?'). The questions were similar to those used in previous studies (e.g., Russell \& Wang, 1997).

Implicit memory was tested using a WSC test. Auditory clips of the word stems were prepared using sound-editing software; each word (i.e., auditory file) was truncated to preserve only the first syllable of the word. A computer was used to present the audio clips of the word stems. The testing set-up was explained to the patients and the computer's screen was positioned to face the patient at a comfortable reading distance. Two practice stems (not from the 80 -word pool) were used to verify satisfactory testing conditions.

Patients were presented with auditory stems of all 80 words from the word pool, of which 20 had been presented as complete words during surgery. The stems were presented in a random order. Each audio clip was played twice, 2 seconds apart. The letters making up each stem appeared on the computer screen at the same time that the stem was heard. However, patients were told that their completion did not need to match the letters they saw but only needed to match the sound that they heard. Patients were instructed to complete the word-stem with the first word that came to mind, but that all could be completed by two-syllable words. Nevertheless, the first response of the patient was recorded as their answer, regardless of whether it was a two-syllable completion. The experimenter was blinded as to which words during test had been presented during the surgery.

Two independent coders subsequently listened to the recordings and transcribed the patients' responses. These coders were blinded to the study hypotheses as well as to which words a particular patient had heard while under anesthesia. In case of a disagreement between the coders, they met to discuss and resolve any transcriptions about which they disagreed.

\section{Results}

BIS values during word presentation in each group are detailed in Figure 1. Average BIS values for the entire priming period in the BIS 40 - 45 and BIS 55 - 60 groups were $39 \pm 5$ and $55 \pm 7$, respectively (mean \pm SD). Average BIS values were significantly different between two groups $(t(36)=8.09$, $\mathrm{p}<0.0001$ ). The two groups also significantly differed in their BIS values at every minute during priming (see Figure 1).

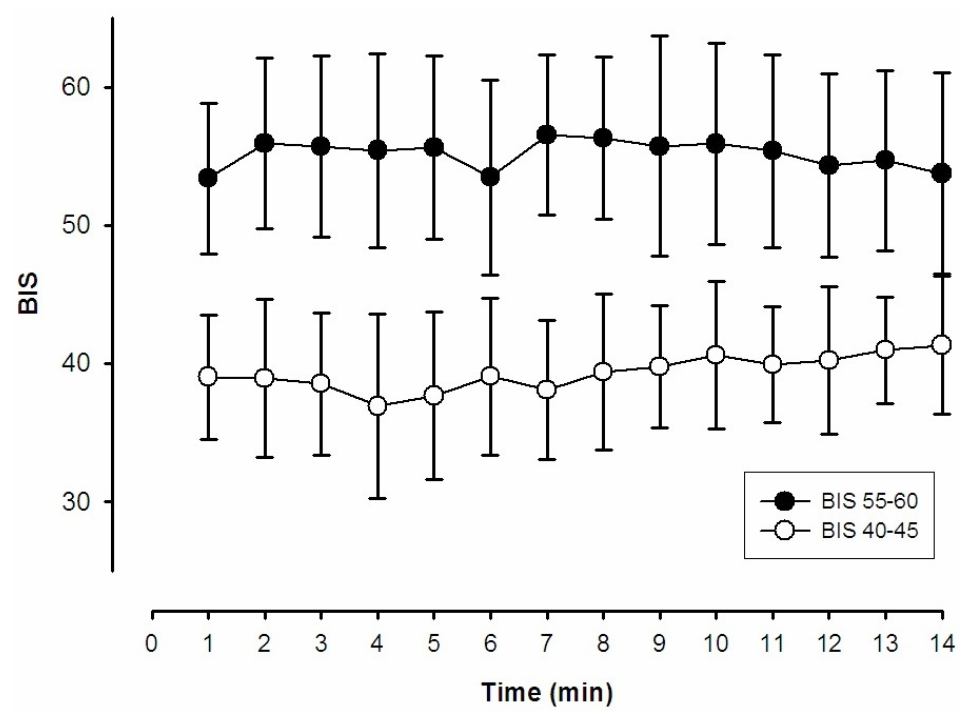

Figure 1.

BIS values during the auditory word presentation in 37 patients. Word presentation started at minute 1 and ended between minutes 14 and 15 . Open $(O)$ and solid $(\bullet)$ circles represent the means for BIS 55 - 60 group $(n=17)$ and BIS 40 - 45 group $(n=20)$, respectively. Error bars represent standard deviations. 
There was no evidence of explicit memory formation during surgery. No patient had any recollection of the intraoperative events when asked the series of free-response questions. For all patients, the last memory before falling asleep was related to the application of monitors prior to anesthesia induction, and the first memory after awakening was being in the recovery room. None of the patients reported dreaming or hearing words during surgery.

The WSC test results were analyzed using a dependent samples $t$-test because patients heard and were tested with both presented and nonpresented word stems. An extreme studentized deviate (ESD) method (Grubbs' test) was used to detect outliers of the individual subjects' WSC test results. When hit rates for individual subjects were analyzed, no outliers were detected. The subject with biggest difference in hit rates for presented and non-presented words was in the BIS $40-45$ group, and had a value of $-0.27(Z=2.29)$. This value was not detected as a significant outlier ( $p>0.05, Z>3.00$ cut-off for significance).

There was no evidence of implicit memory formation. Words that were presented to the patients while under anesthesia were no more likely to be completed during the test phase than words that were not presented (Table 3). No statistical significance was found between words presented and not presented for either BIS level separately or even when combined across BIS level $(\mathrm{t}(36)=-1.62, \mathrm{p}=0.11)$. Note that this marginal effect was in the opposite direction from what would be expected if implicit memories were being formed to the presented words.

Typical statistical tests only allow the null hypothesis to be rejected or not. But sometimes, the null hypothesis is the correct hypothesis and not rejecting it is the correct decision. Rouder et al. (2009) recently developed a Bayes factor (BF) alternative to the conventional $t$-test that allows researchers to express the degree of preference for the null hypothesis. Prior to the availability of this BF methodology, researchers were unable to specify the degree of evidence in support of the null hypothesis. The BF is interpreted as an odds ratio. For example, a BF value of 3 signals that for a set of results, the null is three times as likely to be true as the alternative. To compute the BF, the researcher need only provide the sample size, the $t$-value, and an estimate of the effect size. (Software is available at http://pcl.missouri.edu/bayesfactor.) The researcher must choose an effect size that reflects the paradigm being used. Because the literature shows that the implicit priming effect is small, we used an effect size of $r=0.1$, which assumes that effect sizes of 0.3 or larger are uncommon. This small value of $r$ also is appropriate when small differences are of theoretical importance, which would be the case if information presented while a pa- tient is under anesthesia actually were preserved.

The techniques Rouder et al. (2009) developed for expressing the BF were for two-tailed hypotheses only. However, we cast our alternative hypothesis as a one-tailed test because the predicted result was that WSC in the presented condition would be superior to WSC in the not presented condition. That meant that the null hypothesis was supported if the completion rate in the not presented condition was equal to or better than in the presented condition. For the BIS 40 - 45 condition, the BF was 3.63 (i.e., the null was 3.63 times as likely to be true than the alternative hypothesis). For the BIS 55 - 60 condition, BF was 2.68. A meta-analytic BF, computed by multiplying these two $\mathrm{BF}$ values, was 9.73. In other words, given our data, the null was 9.73 times as likely to be true than the alternative hypothesis. This is strong support against the existence of implicit memory being formed while under anesthesia.

There was no evidence of a greater likelihood for implicit preservation of negative words. The proportion of completions did not differ between the negative versus neutral presented words (negative $=0.20 \pm 0.08$, neutral $=0.28 \pm 0.1$ ) .

\section{Discussion}

We found no evidence for implicit memory at lighter or deeper anesthetic planes in patients undergoing orthopedic procedures. Mean completion rates for presented and non-presented words in the BIS $55-60$ group were $0.24 \pm 0.11$ and $0.26 \pm 0.08$, respectively. In the BIS 40 - 45 group, completion rates for presented and non-presented words were $0.21 \pm 0.15$ and $0.25 \pm$ 0.1 , respectively. In fact, Bayes factor methodology showed that the null hypothesis of "no implicit memory during anesthesia” for all subjects was 9.73 times more likely to be correct than the alternative hypothesis. It is possible that there might be a few subjects demonstrating implicit memory (i.e. outliers), which could have been obscured in an analysis of the whole group. This was ruled out by a Grubbs' test that did not detect any outliers among the study population. Explicit memory also was absent, indicated by the responses given to a standard set of questions.

Our results were consistent with the findings of a recent study by Hadzidiakos et al. (2009) that used a WSC test and an enhanced PDP procedure, neither of which showed an implicit memory effect. In that study, the patients had a median BIS value of 32.5, which corresponded to an anesthetic depth where implicit memory was abolished even in studies with positive findings (e.g., Iselin-Chaves et al., 2005; Stonell et al., 2006). Our findings extend those of Hadzidiakos et al. by showing that implicit memory was absent even at higher BIS ranges.

Table 3.

Proportion of Successfully Completed Words in the WSC Test.

\begin{tabular}{cccc}
\hline & $\begin{array}{c}\text { BIS 40-45 } \\
(n=20)\end{array}$ & $\begin{array}{c}\text { BIS 55-60 } \\
(n=17)\end{array}$ & $\begin{array}{c}\text { Overall } \\
(n=37)\end{array}$ \\
\hline Presented & $0.21 \pm 0.15$ & $0.24 \pm 0.11$ & $0.23 \pm 0.14$ \\
Not Presented & $0.25 \pm 0.10$ & $0.26 \pm 0.08$ & $0.25 \pm 0.09$ \\
\hline
\end{tabular}


Our study had several strengths that support the evidence in favor of the null hypothesis. The main strength was the relative uniformity of the surgical stimulus and depth of anesthesia at the time of word presentation. For all subjects, presentation of the words started within 2 - 3 minutes of bone instrumentation. Depth of anesthesia for each subject was achieved before the word presentation was started and maintained for the duration of priming. To our knowledge, no other study maintained depth of anesthesia in as tight a target interval.

One weakness of the study was that the timing of the memory test was performed on the day of surgery for some subjects but on the next day in others. Ideally, all WSC test should have been conducted after the same time interval because Dobrunz, Jaeger, and Vetter (2007) found a difference in WSC related to timing of the test. It is also possible that the level of the surgical stimulus might not have been strong enough to facilitate implicit learning. It has been proposed that surgical stimulus enables learning during anesthesia. Although there is no direct evidence from human studies, epinephrine has been shown to enable fear conditioning in rats (Gold, Weinberger, \& Sternberg, 1985). The majority of studies that have found implicit memory under anesthesia had ongoing surgical stimulus during the priming phase. Conversely, studies on healthy volunteers under anesthesia in the absence of a surgical stimulus were unable to detect implicit memory. Therefore, it is possible that the level of anesthesia in our study, even in the BIS 55 - 60 group, might have been sufficient to prevent implicit learning given the surgical procedures our subjects were exposed to. We also premedicated our subjects with midazolam, which has been avoided in most other studies. Benzodiazepines are known to have anterograde amnestic properties on the formation of explicit memories, but their effect on implicit memory is not established. Lastly, our methods might be not sensitive enough to detect implicit memory in this setting. Intraoperative word priming followed by a postoperative WSC test as a method of detecting implicit memory under anesthesia is still evolving. Many factors such as timing of the WSC test, choice of target and distractor words, and whether PDP is utilized to remove possible contamination by explicit memories, are still being debated. No method has been established as a standard for testing implicit memory in the perianesthetic state.

In summary, we were unable to demonstrate implicit memory in two groups of patients under different anesthetic depths (BIS 40 - 45 and BIS 55 - 60) during bone instrumentation. According to our results, maintaining the BIS below 60 is an adequate strategy to prevent the formation of implicit as well as explicit memories. This conclusion is 9.73 times more likely to be true than the alternative hypothesis that implicit memories can be formed under anesthesia.

\section{Acknowledgements}

We gratefully acknowledge the support of the Vice President for Research from the University of Oklahoma for this project. We would like to thank Jeffrey N. Rouder and Michael S. Pratte for their assistance with the statistical analysis.

\section{References}

Andrade, J., \& Deeprose, C. (2007). Unconscious memory formation during anesthesia. Best Practices and Research Cliicaln Anesthesiology, 21, 385-401. doi:10.1016/j.bpa.2007.04.006
Bowdle, T. A. (2006). Depth of anesthesia monitoring. Anesthesiology Clinics of North America, 24, 793-822. doi:10.1016/j.atc.2006.08.006

Deeprose, C., Andrade, J., Varma, S., \& Edwards, N. (2004). Unconscious learning during surgery with propofol anesthesia. British Journal of Anesthesia, 92, 171-177. doi:10.1093/bja/aeh054

Dobrunz, U. E., Jaeger, K., \& Vetter, G. (2007). Memory priming during light anesthesia with desflurane and remifentanil anesthesia. British Journal of Anesthesia, 98, 491-496. doi:10.1093/bja/aem008

Gold, P. E., Weinberger, N. M., \& Sternberg, D. B. (1985). Epinephrine-induced learning under anesthesia: Retention performance at several training-testing intervals. Behavioral Neuroscience, 99, 1019-1022. doi:10.1093/bja/aem008

Hadzidiakos, D. A., Horn, N., Degener, R., Buchner, A., \& Rehberg, B. (2009). Analysis of memory formation under general anesthesia (propofol/remifentanil) for elective surgery using the process-dissociation procedure. Anesthesiology, 111, 293-301. doi:10.1097/ALN.0b013e3181ac4a4b

Iselin-Chaves, I. A., Willems, S. J., Jermann, F. C., Forster, A., Adam, S. R., \& Van der Linden, M. (2005). Investigation of implicit memory during isoflurane anesthesia for elective surgery using the process dissociation procedure. Anesthesiology, 103, 925-933. doi:10.1097/00000542-200511000-00005

Jacoby, L. L. (1991). A process dissociation framework: Separating automatic from intentional uses of memory. Journal of Memory and Language, 30, 513-541. doi:10.1016/0749-596X(91)90025-F

John, C. H. (1988). Emotionality ratings and free association norms of 240 emotional and non-emotional words. Cognition and Emotion, 2 , 49-70. doi:10.1080/02699938808415229

Kerssens, C., Ouchi, T., \& Sebel, P. S. (2005). No evidence of memory function during anesthesia with propofol or isoflurane with close control of hypnotic state. Anesthesiology, 102, 57-62. doi:10.1097/00000542-200501000-00012

Lequeux, P. Y., Cantraine, F., Levarlet, M., \& Barvais, L. (2003). Absence of explicit and implicit memory in unconscious patients using a TCI of propofol. Acta Anaesthesiologica Scandinavica, 47, 833-837. doi:10.1034/j.1399-6576.2003.00159.X

Lequeux, P. Y., Velghe-Lenelle, C. E., Cantraine, F., Sosnowski, M., \& Barvais, L. (2005). Absence of implicit and explicit memory during propofol/remifentanil anesthesia. European Journal of Anaesthesiology, 22, 333-336. doi:10.1017/S0265021505000566

Lubke, G. H., Kerssens, C., Phaf, H., \& Sebel, P. S. (1999). Dependence of explicit and implicit memory on hypnotic state in trauma patients. Anesthesiology, 90, 670-680. doi:10.1097/00000542-199903000-00007

Munte, S., Munte, T. F., Mitzlaff, B., Walz, R., Leuwer, M., \& Piepenbrock, S. (2001). Postoperative reading speed does not indicate implicit memory in elderly cardiac patients after propofol and remifentanyl anesthesia. Acta Anaesthesiologica Scandinavica, 45, 750-755. doi:10.1034/j.1399-6576.2001.045006750.x

Phelps, E. A. (2004). Human emotion and memory: Interactions of the amygdala and hippocampal complex. Current Opinion in Neurobiology, 14, 198-202. doi:10.1016/j.conb.2004.03.015

Rouder, J. N., Speckman, P. L., Sun, D., Morey, R. D., \& Iverson, G. (2009). Bayesian $t$-tests for accepting and rejecting the null hypothesis. Psychonomic Bulletin and Review, 16, 225-237. doi:10.3758/PBR.16.2.225

Russell, I. F., \& Wang, M. (1997). Absence of memory for intraoperative information during surgery under adequate general anesthesia. British Journal of Anesthesia, 78, 3-9.

Russell, I. F., \& Wang, M. (2001). Absence of memory for intra-operative information during surgery with total intravenous anesthesia. British Journal of Anesthesia, 86, 196-202. doi:10.1093/bja/86.2.196

Sandin, R. (2006). Outcome after awareness with explicit recall. Acta Anaesthesiologica Belgica, 57, 429-432.

Schacter, D. L. (1987). Implicit memory: History and current status. Journal of Experimental Psychology: Learning, Memory, and Cognition, 13, 501-518. doi:10.1037/0278-7393.13.3.501

Stoelting, R. K. (1999). APSF survey results identify safety issue priorities. APSF Newsletter 1999, 14, 2. 
Stoelting, R. K. (1999). APSF survey results identify safety issue priorities. APSF Newsletter, Spring, 14, 5-6.

Stonell, C. A., Leslie, K., He, C., \& Lee, L. (2006). No sex differences in memory formation during general anesthesia. Anesthesiology, 105,
920-926. doi:10.1097/00000542-200611000-00012

Wright, A. J., \& Aldrete, A. (1987). Patient memories of anesthesia-historical perspective. Middle East Journal of Anesthesiology, 9, 233-254. 